\title{
EXISTENCE TESTS FOR LIMITING CYCLES OF SECOND ORDER DIFFERENTIAL EQUATIONS
}

\author{
M.K. ARABOV, E. MUKHAMADIEV, I.D. NUROV, Kh.I. SOBIROV
}

\begin{abstract}
This work is devoted to finding limiting cycles in the vicinity of equilibria of second order nonlinear differential equations. We obtain new conditions for the coefficients of the equations ensuring the existence of a limiting cycle by employing the methods of qualitative analysis and computer modeling. We study the behavior of a singular point under variation of the parameters and we apply the Lyapunov stability theory. On the base of the obtained results, we make a sector partition of the plane. This partition allows us to predict the behavior of the solutions in various parts of the plane. We develop a package of computer programs for constructing a phase portrait in the corresponding domains.
\end{abstract}

Ключевые слова: dynamical systems, nonsmoothness, phase portraits, limiting cycles, sectorial partitions.

\section{Mathematics Subject Classification: $34 \mathrm{E}$}

Limiting cycles have wide applications in many fields in natural sciences: in radiophysics, automatic control, chemistry, medicine, mathematical biology, economics, etc. This is why studying the existences of limiting cycles is an important part of the theory of nonlinear oscillations. Various sufficient conditions for the existence of limiting cycles are known. The classical examples of the equations with limiting cycles are Van der Pol equations and Rayleigh equation [1-6]. Rather recently there was found that piecewise linear equations of the form

$$
x^{\prime \prime}+a x^{\prime}+b x+c\left|x^{\prime}-\lambda\right|=0
$$

possess limiting cycles for certain values of the coefficients $a, b, c$ and of the parameter $\lambda$. Namely, by means of the computer modelling, it was established in work [7] that equation (1) with $a=1, b=1, c=3 / 2$ and $\lambda>0$ has a limiting cycle. A more general existence condition for a limiting cycle of equation (1) was obtained in work [8].

As many authors mention, see, for instance, [3], the existence of the limiting cycles is one of the most complicated issues and there are no general approaches for solving this. This is why any method allowing to establish the existence of a limiting cycle at least partially is of interest.

In the present work we study a nonlinear second order differential equation

$$
x^{\prime \prime}+a x^{\prime}+b x+c\left|x^{\prime}-\varphi\left(x, x^{\prime}\right)\right|=0,
$$

where $a, b, c$ are real numbers and the function $\varphi(x, y)$ is continuous and satisfies certain growth condition as $|x|+|y| \rightarrow \infty$. We find the conditions of the coefficients $a, b, c$ and the function $\varphi(x, y)$ ensuring the existence of a limiting cycle for equation (2).

M.K. Arabov, E. Mukhamadiev, I.D. Nurov, Kh.I. Sobirov, Existence tests for limiting Cycles OF SECOND ORDER DIFFERENTIAL EQUATIONS.

(C)Arabov M.K., Mukhamadiev E.M., Nurov I.D., Sobirov Kh.I. 2017.

Submitted April 19, 2016. 


\section{ANALYSIS OF THE PHASE PORTRAIT FOR HOMOGENEOUS EQUATION}

We begin by analysing the phase portrait of the homogeneous equation

$$
x^{\prime \prime}+a x^{\prime}+b x+c\left|x^{\prime}\right|=0
$$

subject to the location of the coefficients $(a, b, c)$ as a point in the three-dimensional space $\mathbb{R}^{3}$, see [8]. If $c=0$, then (3) is a linear second order equation, whose phase portrait is well-known, see, for instance, $[1,2,4]$. In what follows we assume that $c>0$. We observe that if $c<0$, the change of $x$ by $-x$ transforms equation (3) to the same equation with the coefficients $(a, b,-c)$.

Equation (3) is "glued" by the linear equations

$$
x^{\prime \prime}+(a+c) x^{\prime}+b x=0, \quad \text { if } \quad x^{\prime}>0
$$

and

$$
x^{\prime \prime}+(a-c) x^{\prime}+b x=0, \quad \text { if } \quad x^{\prime} \leqslant 0 .
$$

By $\mu_{1}^{ \pm}$and $\mu_{2}^{ \pm}$we denote the roots of the characteristic equation

$$
\mu^{2}+(a \pm c) \mu+b=0
$$

corresponding to equations (4) and (5):

$$
\mu_{1}^{ \pm}=-\frac{1}{2}\left\{(a \pm c)-\sqrt{(a \pm c)^{2}-4 b}\right\}, \quad \mu_{2}^{ \pm}=-\frac{1}{2}\left\{(a \pm c)+\sqrt{(a \pm c)^{2}-4 b}\right\} .
$$

These formulae for the roots of characteristic equations (6) yield that in the half-space $\{(a, b, c)$ : $c>0\}$ of the coefficients of equation (3), the roots $\mu_{1}^{ \pm}$vanish as $b=0$ and change the sign as $b$ grows. The roots $\mu_{1}^{ \pm}, \mu_{2}^{ \pm}$are respectively real as $4 b<(a \pm c)^{2}$, are degenerate as $4 b=(a \pm c)^{2}$ and complex conjugate as $4 b>(a \pm c)^{2}$.

In view of the above properties of the roots of the characteristic equations, we partition the half-space $\{(a, b, c): c>0\}$ into the following subsets:

1. $\{(a, b, c): b<0\}$;

2. $\left\{(a, b, c): 0<4 b \leqslant(|a|-c)^{2},|a|>c\right\}$;

3. $\left\{(a, b, c): 0<4 b \leqslant(|a|-c)^{2},|a|<c\right\}$;

4. $\left\{(a, b, c):(|a|-c)^{2}<4 b \leqslant(|a|+c)^{2}\right\}$;

5. $\left\{(a, b, c):(|a|+c)^{2}<4 b\right\}$.

The projection on the coordinate plane $(a, b)$ of the intersections of these subsets with the plane $c=$ const $>0$ is shown on Fig. 1 .

We observe that for the coefficients $(a, b, c)$ in sets $1-4$ the non-zero solution $x(t)$ to equation (3) with the initial conditions $x\left(t_{0}\right)=x_{0}, x^{\prime}\left(t_{0}\right)=0$ is a solution to linear equation (4) as $\left(t-t_{0}\right) x_{0}<0$ to equation (5) as $\left(t-t_{0}\right) x_{0}>0$.

Equation (3) is equivalent to the system

$$
\left\{\begin{array}{l}
x^{\prime}=y, \\
y^{\prime}=-a y-b x-c|y| .
\end{array}\right.
$$

If $b \neq 0$, then system $(7)$ has the only singular point $(0,0)$. As for a linear equation, the location of the roots of characteristic equations (6) on the complex plane determines uniquely the behavior of the trajectories of piecewise linear system (7) in the phase plane $(x, y)$. As we see in the table given below, the piecewise linear systems have isolated complicated singular points, where there arises an elliptic sector formed by the trajectories approaching the singular point both as $t \rightarrow \infty$ and $t \rightarrow-\infty$.

In what follows we assume that $b \neq 0$. In the following table we classify the main cases of the behavior of the trajectories of system (7) on the phase plane. 


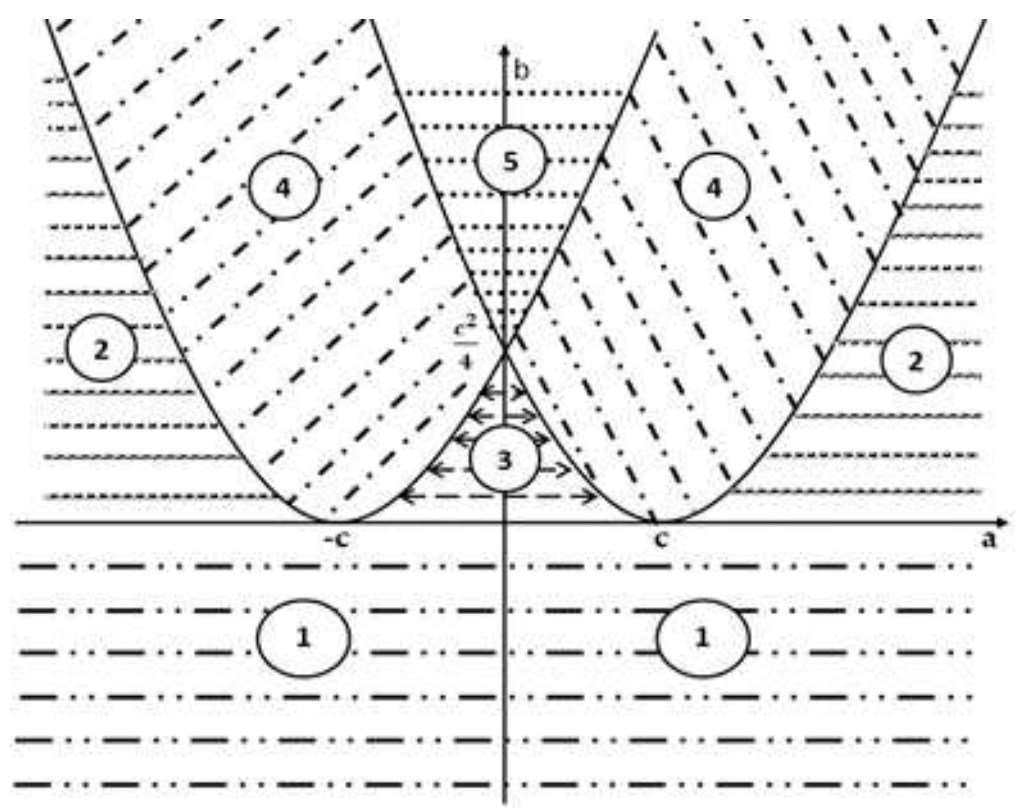

FiguRE 1. Sectors

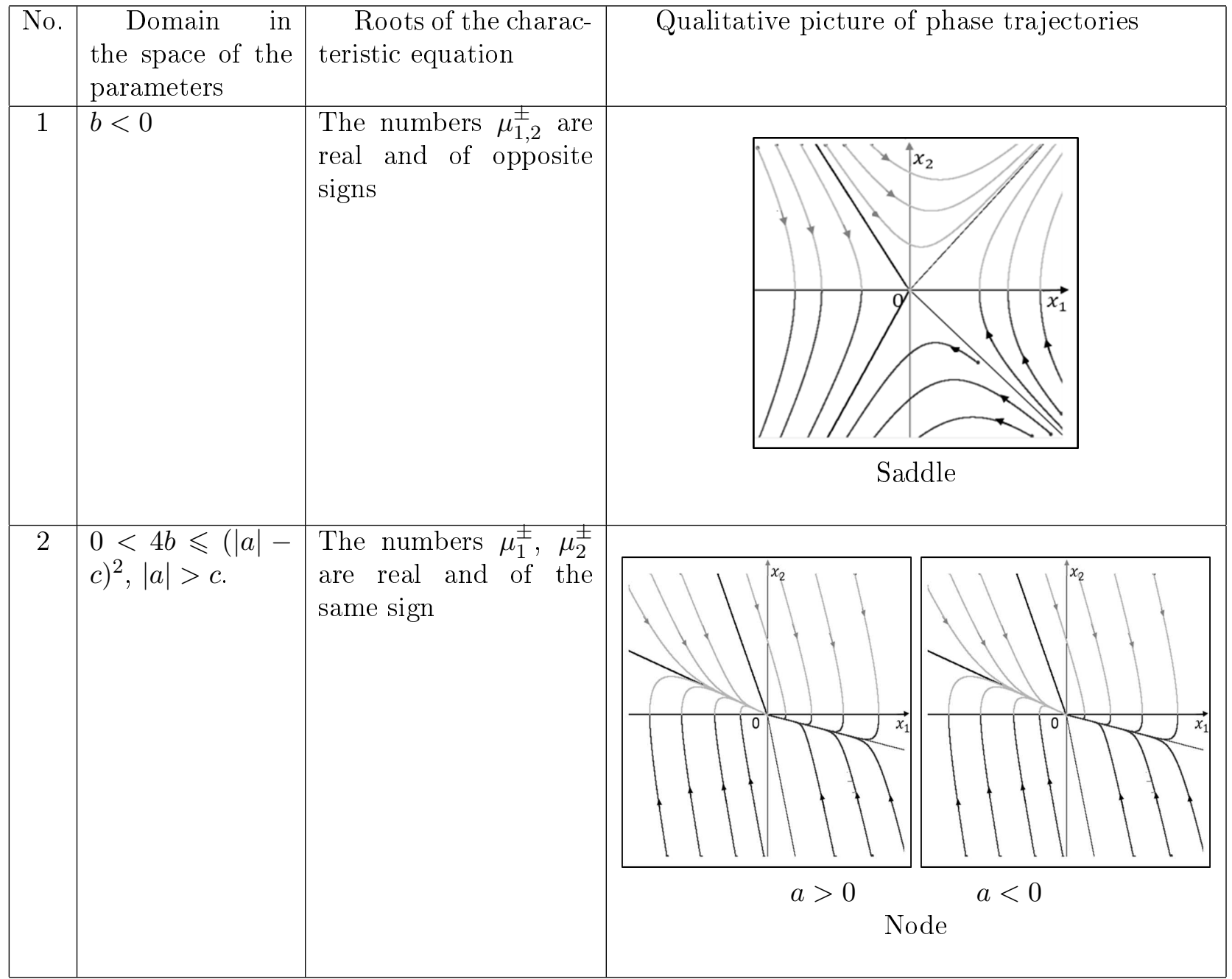




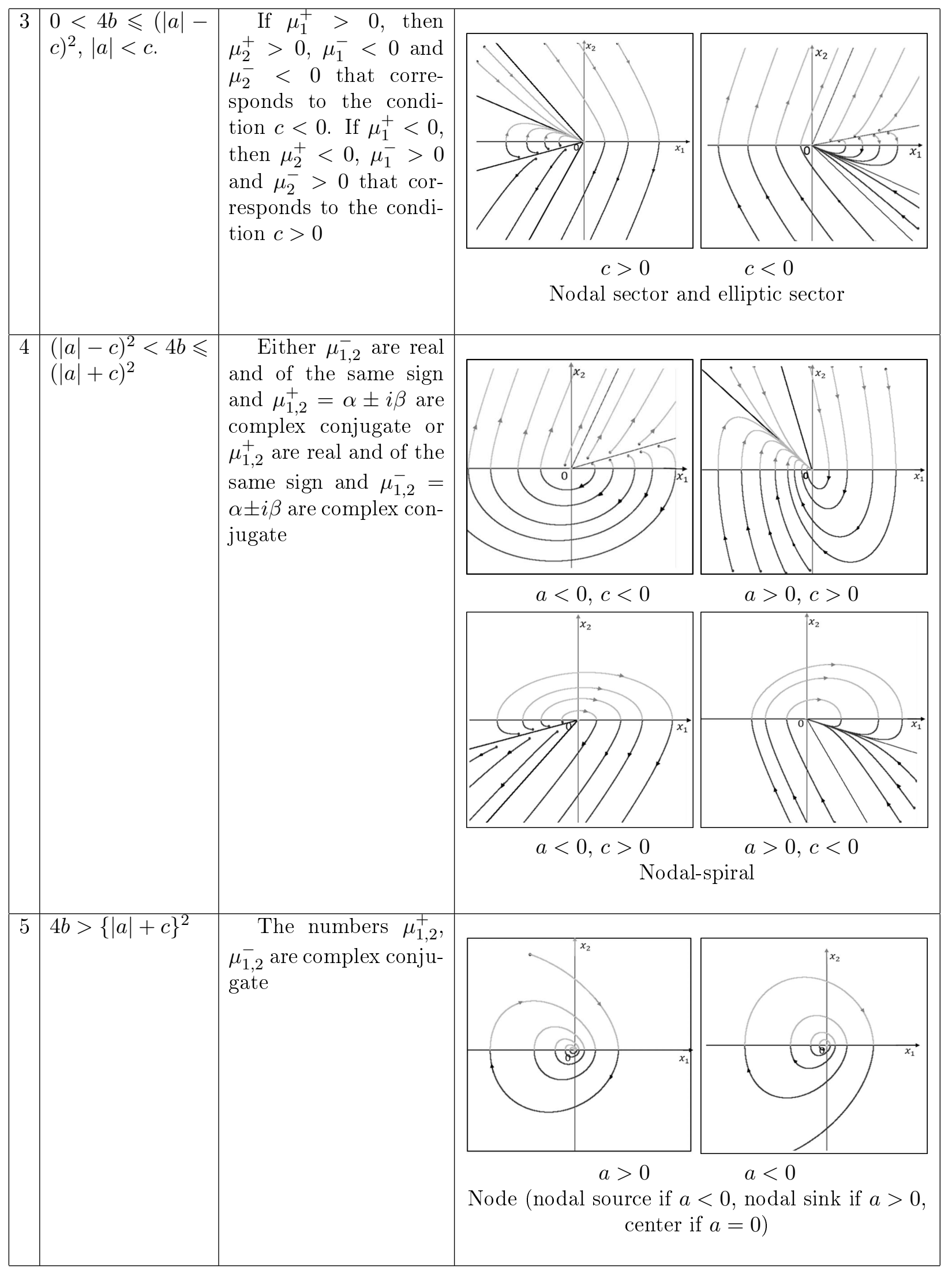

\section{CONDITIONS FOR BOUNDEDNESS OF SOLUTION ON HALF-LINE}

We proceed to studying equation (2). Equation (2) is equivalent to the system 


$$
\left\{\begin{array}{l}
x^{\prime}=y, \\
y^{\prime}=-a y-b x_{1}-c|y-\varphi(x, y)| .
\end{array}\right.
$$

In what follows we assume that the function $\varphi(x, y)$ satisfies the condition

$$
\lim _{|x|+|y| \rightarrow \infty} \frac{|\varphi(x, y)|}{|x|+|y|}=0 .
$$

Condition (9) guarantees the possibility to extend the solution of system (8) on the entire line $(-\infty, \infty)$.

Theorem 1. Assume that (9) holds and the coefficients of system (8) satisfies the conditions

$$
b>0, \quad c>0, \quad a \notin[\min \{0,2 \sqrt{b}-c\}, \max \{0, c-2 \sqrt{b}\}] .
$$

Then all solutions of system (8) are bounded as $t>0$ if $a>0$ and as $t<0$ if $a<0$, that is, $a \neq 0$ and for each solution $(x(t), y(t))$ the inequality

$$
\sup \{|x(t)|+|y(t)|: a \cdot t>0\}<\infty .
$$

holds true.

Proof. Let $a>0$. If some solution $(x(t), y(t))$ of system (8) is unbounded as $t>0$, then there exists a sequence $t_{k}, 0<t_{k}<t_{k+1}, k=1,2, \ldots$, such that $t_{k} \rightarrow \infty$ and $\left|x\left(t_{k}\right)\right|+\left|y\left(t_{k}\right)\right| \rightarrow \infty$ as $k \rightarrow \infty$. We choose numbers $\tau_{k} \in\left[0, t_{k}\right]$ such that

$$
\left|x\left(\tau_{k}\right)\right|+\left|y\left(\tau_{k}\right)\right|=\max _{0 \leqslant t \leqslant t_{k}}(|x(t)|+|y(t)|) .
$$

The inequality $\left|x\left(\tau_{k}\right)\right|+\left|y\left(\tau_{k}\right)\right| \geqslant\left|x\left(t_{k}\right)\right|+\left|y\left(t_{k}\right)\right|$ implies that $d_{k} \equiv\left|x\left(\tau_{k}\right)\right|+\left|y\left(\tau_{k}\right)\right| \rightarrow \infty$ and therefore, $\tau_{k} \rightarrow \infty$ as $k \rightarrow \infty$. The vector functions

$$
\left(u_{k}(t), v_{k}(t)\right)=\left(x\left(\tau_{k}+t\right) / d_{k}, y\left(\tau_{k}+t\right) / d_{k}\right) \quad-\tau_{k} \leqslant t \leqslant 0
$$

satisfy the conditions

$$
\left|u_{k}(t)\right|+\left|v_{k}(t)\right| \leqslant\left|u_{k}(0)\right|+\left|v_{k}(0)\right|=1, \quad-\tau_{k} \leqslant t \leqslant 0
$$

and solve the system

$$
\left\{\begin{array}{l}
u^{\prime}=v, \\
v^{\prime}=-a v-b u-c\left|v-h_{k}(t)\right| .
\end{array}\right.
$$

Here the functions $h_{k}(t)$ are determined by the identity $h_{k}(t)=\varphi\left(u_{k}(t), v_{k}(t)\right) / d_{k}$ and by the choice of the numbers $d_{k}$ and condition (9), they tend to zero as $k \rightarrow \infty$ uniformly in $t$.

Let $\left(u^{*}, v^{*}\right)$ be the limiting point of the sequence $\left(u_{k}(0), v_{k}(0)\right)$. Then the solution to system (7) satisfying the initial condition $(x(0), y(0))=\left(u^{*}, v^{*}\right)$ is non-zero and bounded as $t \leqslant 0$. On the other hand, under the assumptions of the theorem and according lines 2 and 4 in the table, all solutions to system (7) are unbounded as $t \leqslant 0$. The obtained contradiction proves the theorem for $a>0$.

The case $a<0$ is considered in the same way.

\section{STABILITY OF ISOLATED SINGULAR POINT}

We consider a general second order equation

$$
x^{\prime \prime}=f\left(x, x^{\prime}\right) \text {, }
$$

where $f(x, y)$ is a continuous function on the entire plane $(x, y)$. 
Equation 111 is equivalent to the system of equations

$$
\left\{\begin{array}{l}
x^{\prime}=y, \\
y^{\prime}=f(x, y) .
\end{array}\right.
$$

In what follows we assume that each solution to system 12 can be extended to the entire real line. As we have mentioned above, this assumption is true in the particular case of equation (8), where the function $\varphi(x, y)$ satisfies condition (9).

Singular points of system (12) are located on the abscisse axis, that is, they are of the form $\left(x_{0}, 0\right)$, where $x_{0}$ is a solution to the scalar equation

$$
f(x, 0)=0 .
$$

Let $x_{0}$ be a solution of scalar equation (13). It should be noted that studying the behavior of trajectories of system (12) in the vicinity of equilibria is one of the main issues in the qualitative theory of differential equations.

In what follows we shall need some properties related to the qualitative behavior of the trajectories of system (12) in the vicinity of a singular point $\left(x_{0}, 0\right)$. In order to describe these properties, we employ the Lyapunov function [2,3].

Lemma 1. Assume that in some neighbourhood $\left|x-x_{0}\right|+|y|<\sigma, \sigma>0$, of a singular point $\left(x_{0}, 0\right)$ the function $f(x, y)$ satisfies the conditions:

a) $f(x, 0)\left(x-x_{0}\right) \leqslant 0$ and $f(x, 0)$ is not identically zero in each interval $(-\delta, 0)$ and $(0, \delta)$, $\delta>0$;

b) $(f(x, y)-f(x, 0)) y \geqslant 0$.

Then for each solution $(x(t), y(t))$ to system (12) not coinciding with the stationary solution $\left(x_{0}, 0\right)$ the inequality

$$
\inf _{t \geqslant 0}\left[\left|x(t)-x_{0}\right|+|y(t)|\right]>0
$$

holds true.

Proof. Suppose that the statement of the lemma is wrong. Then there exists a solution $(x(t), y(t))$ to system (12) not coinciding with the singular point $\left(x_{0}, 0\right)$ and a sequence $t_{1}<t_{2}<\ldots<t_{k}<\ldots$ such that

$$
\lim _{k \rightarrow \infty}\left|x\left(t_{k}\right)-x_{0}\right|+\left|y\left(t_{k}\right)\right|=0 .
$$

We consider the set

$$
E=\left\{t: t \geqslant 0,\left|x(t)-x_{0}\right|+|y(t)|<\sigma_{1}\right\}
$$

where

$$
0<\sigma_{1}<\min \left\{\sigma,\left|x(0)-x_{0}\right|+|y(0)|\right\} .
$$

We observe that $E$ is an open set and by (15), $t_{k} \in E$ starting from some number $k_{0}$. By $\left(\alpha_{k}, \beta_{k}\right)$ we denote the intervals in the set $E$ containing the numbers $t_{k}$. It is possible that the intervals $\left(\alpha_{k}, \beta_{k}\right)$ corresponding to different indices $k$ coincide $\beta_{k}=\infty$ starting from some index $k$. By the definition of an interval in the set $E$ we have

$$
\left|x(t)-x_{0}\right|+|y(t)|<\left|x\left(\alpha_{k}\right)-x_{0}\right|+\left|y\left(\alpha_{k}\right)\right|=\sigma_{1}, \quad \alpha_{k}<t \leqslant t_{k} .
$$

The solution $(x(t), y(t))$ satisfies the identity

$$
\frac{d}{d t}\left(\frac{y^{2}}{2}+G(x)\right)=h(x, y)
$$

where

$$
G(x)=-\int_{x_{0}}^{x} f(s, 0) d s, \quad h(x, y)=(f(x, y)-f(x, 0)) y
$$


Integrating identity 17$)$ over the segment $\left[0, t_{k}\right]$, we get

$$
\left.\frac{y^{2}\left(t_{k}\right)}{2}+G\left[x\left(t_{k}\right)\right]-\frac{y^{2}\left(\alpha_{k}\right)}{2}-G\left[\alpha_{k}\right)\right]=\int_{\alpha_{k}}^{t_{k}} h(x(s), y(s)) d s .
$$

By Condition b), $h(x, y) \geqslant 0$ as $\left|x-x_{0}\right|+|y|<\sigma$ and this is why the integrand in the right hand side of 18 is non-negative. Therefore, the inequality

$$
\left.\frac{y^{2}\left(t_{k}\right)}{2}+G\left[x\left(t_{k}\right)\right]-\frac{y^{2}\left(\alpha_{k}\right)}{2}-G\left[\alpha_{k}\right)\right] \geqslant 0, k \geqslant k_{0}
$$

holds true. Since by Condition a) the continuous function $G(x)$ is positive as $0<\left|x-x_{0}\right|<\sigma$, then $\frac{y^{2}}{2}+G[x] \geqslant m>0$ as $\left|x-x_{0}\right|+|y|=\sigma_{1}$. This is inequality 19 contradicts identity (15). This contradiction completes the proof.

We observe that if the function $f(x, y)$ has continuous partial derivatives $\frac{\partial f}{\partial x}(x, y), \frac{\partial f}{\partial y}(x, y)$ in the vicinity of a singular point $\left(x_{0}, 0\right)$, in order to satisfy Conditions a) and b) of Lemma 1 , it is sufficient to assume the inequalities $\frac{\partial f}{\partial x}\left(x_{0}, 0\right)<0, \frac{\partial f}{\partial y}\left(x_{0}, 0\right)>0$.

The next lemma generalizes Lemma 1.

Lemma 2. Assume that in some neighbourhood $\left|x-x_{0}\right|+|y|\langle\sigma, \sigma>0$ of a singular point $\left(x_{0}, 0\right)$ and for a given $\kappa \leqslant 0$ the function $f(x, y)$ satisfies the conditions

a) the function $f\left(x, \kappa\left(x-x_{0}\right)\right)\left(x-x_{0}\right)-\kappa^{2}\left(x-x_{0}\right)^{2} \geqslant 0$ is not identically zero in each interval $(-\delta, 0)$ and $(0, \delta), \delta>0$;

b) $\left(f\left(x, \kappa\left(x-x_{0}\right)+y\right)-\kappa y-f\left(x, \kappa\left(x-x_{0}\right)\right)\right) y \geqslant 0$.

Then for each solution $(x(t), y(t))$ of system 12) not coinciding with the stationary solution $\left(x_{0}, 0\right)$ the inequality

$$
\inf _{t \geqslant 0}\left(\left|x(t)-x_{0}\right|+|y(t)|\right)>0
$$

holds true.

Similar to Lemma 1, the following statement can be proved.

Lemma 3. Assume that in some neighbourhood $\left|x-x_{0}\right|+|y|<\sigma, \sigma>0$, of a singular point $\left(x_{0}, 0\right)$ the function $f(x, y)$ satisfies Condition a) of Lemma 1 and the condition

c) $(f(x, y)-f(x, 0)) y \leqslant 0$.

Then for each solution $(x(t), y(t))$ of system (12) not coinciding with the stationary solution $\left(x_{0}, 0\right)$ the inequality

$$
\inf _{t \leqslant 0}\left(\left|x(t)-x_{0}\right|+|y(t)|\right)>0 \text {. }
$$

holds true.

Property (21) of solutions to system (12) is closely related to the Lyapunov stability of the stationary solution $\left(x_{0}, 0\right)$. Namely, the following theorem holds.

Theorem 2. The stationary solution $\left(x_{0}, 0\right)$ of system (12) is Lyapunov stable if and only if each solution not coinciding with this stationary solution satisfies inequality (21).

\section{EXISTENCE TESTS FOR LIMITING CYCLES}

We continue studying system (8) assuming that $b>0, c>0$, the function $\varphi(x, y)$ satisfies condition (9) and $\varphi(0,0)>0$ and system (8) has the only singular point, that is, the scalar equation

$$
b x+c|\varphi(x, 0)|=0
$$

has the only solution $x_{0}$ and $x_{0} \neq 0$. 
Theorem 3. Assume that

a) the coefficients of equation (2) satisfy conditions $a>\max \{0, c-2 \sqrt{b}\}$;

b) in some neighbourhood $\left|x-x_{0}\right|+|y|<\sigma, \sigma>0$ of the singular point $\left(x_{0}, 0\right)$ the function $\varphi(x, y)$ satisfies the inequality $((c-a) y+c \varphi(x, y)-c \varphi(x, 0)) y \geqslant 0$.

Then for each non-stationary solution $(x(t), y(t))$ to system (8) and each sequence $h_{k} \rightarrow+\infty$ there exists a subsequence $h_{k_{j}}$ such that the solutions $\left(x\left(t+h_{k_{j}}\right), y\left(t+h_{k_{j}}\right)\right)$ approach some periodic solution of system (8) as $j \rightarrow+\infty$ uniformly on each segment.

Proof. Let $(x(t), y(t))$ be an arbitrary non-stationary and non-periodic solution to system (8). By Theorem 1, this solution is bounded as $t>0$. Therefore, for each sequence $h_{k} \rightarrow+\infty$, the sequence of the solutions $\left(x\left(t+h_{k}\right), y\left(t+h_{k}\right)\right)$ is well-defined, continuous and equicontinuous on each segment. By Arzelà-Ascoli theorem, there exists a subsequence $h_{k_{j}}$ such that the solutions $\left(x_{1}\left(t+h_{k_{j}}\right), x_{2}\left(t+h_{k_{j}}\right)\right)$ approach some solution $\left(x^{*}(t), y^{*}(t)\right)$ of system $(8)$ uniformly on each segment as $j \rightarrow+\infty$.

By Lemma 1, the set of $\omega$-limiting points of solution $(x(t), y(t))$ does not contain the unique singular point of system (8). This is why by Poincaré-Bendixson theorem [9], the solution $\left(x_{1}^{*}(t), x_{2}^{*}(t)\right)$ is periodic. The proof is complete.

Figure 2 demonstrates the statement of the theorem.

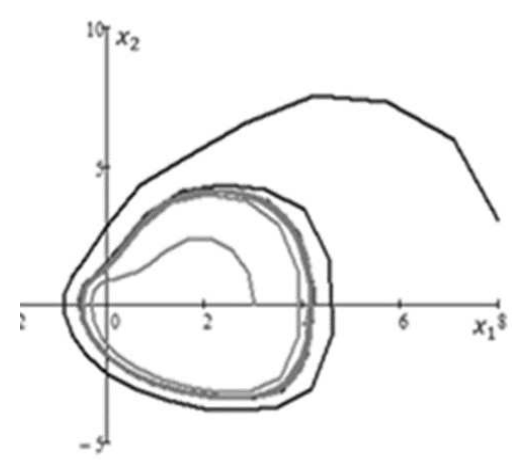

FIGURE 2. Limiting cycle

Theorem 4. Assume that

a) the coefficients of equation (2) satisfy the conditions $a<\min \{0,2 \sqrt{b}-c\}$;

b) in some neighbourhood $\left|x-x_{0}\right|+|y|<\sigma, \sigma>0$, of a singular point $\left(x_{0}, 0\right)$ the function $\varphi(x, y)$ satisfies the inequality $((c-a) y+c \varphi(x, y)-c \varphi(x, 0)) y \leqslant 0$.

Then for each non-stationary solution $(x(t), y(t))$ of system (8) and each sequence $h_{k} \rightarrow+\infty$ there exists a subsequence $h_{k_{j}}$ such that the solutions $\left(x\left(t-h_{k_{j}}\right), y\left(t-h_{k_{j}}\right)\right)$ approach some periodic solution to system (8) as $j \rightarrow+\infty$ uniformly on each segment.

As an example we consider the function $\varphi(x, y)=\cos (x+y)$. It satisfies condition (9) and $\varphi(0,0)=1>0$. The next statement provides a uniqueness condition for solutions to equation (22).

Lemma 4. The equation $d x+|\cos (x)|=0$, where $d$ is a given number, has the unique solution if and only $|d| \sqrt{1+x_{1}^{2}}>1$, where $x_{1} \in(\pi / 2, \pi)$ is the solution to equation $\cos (x)+$ $x \sin (x)=0,\left(x_{1} \approx 2,798386, \sqrt{1+x_{1}^{2}} \approx 0,336508\right)$.

We observe that in the case $|d|>1$, Lemma 4 follows the principle of contracting mappings, while in the case $1<|d| \sqrt{1+x_{1}^{2}} \leqslant \sqrt{1+x_{1}^{2}}$ it is implied by the analysis of the graph of the functions $z=-|\cos (x)|$ and of the straight line $z=d x$. 
Assume that the coefficients $b, c$ are such that $0<c<b \sqrt{1+x_{1}^{2}}$. Then according Lemma 4 , the equation $b x+c|\cos (x)|=0$ has the unique solution $x_{0}=x_{0}(b / c)<0$ depending on the fraction $b / c$. This is why, if the coefficients $a, b, c$ of system (8) satisfies the inequalities $\max \{0, \quad c-2 \sqrt{b}\}<a<c\left(1-\sin \left(x_{0}\right)\right)$, then all assumptions of Theorem 3 are satisfied as $\varphi(x, y)=\cos (x+y)$. Therefore, equation (2) has the limiting cycle.

\section{BIBLIOGRAPHY}

1. V.I. Arnol'd. Geometrical methods in the theory of ordinary differntial equations. Regular and Chaotic Dynamics, Moscow-Izhevsk (2000). [Grundlehren der Mathematischen Wissenschaften. 250. Springer, New York (1988).]

2. A.F. Fillipov. Introduction into theory of differential equations. URSS, Moscow (2004). (in Russian).

3. N.N. Bautin, E.A. Leontovich. Methods and approaches of qualitative study of dynamical systems on plane. Nauka, Moscow (1976). (in Russian).

4. M.G. Yumagulov. Introduction into theory of dynamical systems: handbook. Lan Publishing, St.Petersburg (2015). (in Russian).

5. A.P. Ivanov. Analysis of discontinuous bifurcations in nonsmooth dynamical systems // Nelin. Dinam. 8:2, 231-247 (2012). (in Russian).

6. A.B. Katok, B. Hasselblatt. Introduction to the modern theory of dynamical systems. Cambridge Univ. Press, Cambridge (1997).

7. R.I. Leine, D.H. Van Campen. Bifurcation phenomena in non-smooth dynamical systems // Eur. J. Mech. A/Solids. 25:4, 595-616 (2006).

8. E.M. Mukhamsdiev, I.D. Nurov, M.Sh. Khalilova. Limiting cycles of piece-linear second order differential equations // Ufimskij Matem. Zhurn. 6:1, 84-93 (2014). [Ufa Math. J. 6:1, 80-89 (2014).]

9. Ph. Hartman. Odinary differential equations. John Wiley \& Sons, (1964).

Mullosharaf Kurbonovich Arabov,

Institute of Mathematics named after A. Dzhuraev,

Academy of Sciences of Republic of Tadjikistan,

Aini str. 299/1,

734063 Dushanbe, Tadzhikistan

E-mail: cool.araby@mail.ru

Ergashboy Mirzoevich Mukhamadiev,

Vologda State University,

Lenin str., 15,

160000, Vologda, Russia

E-mail: emuhamadiev@rambler.ru

Iskhokboi Dzhumaevich Nurov,

Tadjik National University,

Rudaki av. 17,

734000, Dushanbe, Tajikistan

E-mail: nid1@mail.ru

Khurshed Ilkhomiddinovich Sobirov,

Tadjik National University,

Rudaki av. 17,

734000, Dushanbe, Tajikistan

E-mail: hurshed.sobirov@mail.ru 